\title{
Transmission characteristics of EM wave in a finite thickness plasma
}

\author{
Nai-Yi Zhu · Li-Shun Huang · Bin Wu · Xi-Long Yu • Run-Hui Wu • Gang Meng · Ai-Min Ren · Xin-Tian Hu
}

Received: 12 December 2012 / Accepted: 6 January 2013

(C)The Chinese Society of Theoretical and Applied Mechanics and Springer-Verlag Berlin Heidelberg 2013

\begin{abstract}
One of the key factors for solving the problems of re-entry communication interruption is electromagnetic (EM) wave transmission characteristics in a plasma. Theoretical and experimental studies were carried out on specific transmission characteristics for different plasma sheath characteristic under thin sheath condition in re-entry state. The paper presents systematic studies on the variations of wave attenuation characteristics versus plasma sheath thickness $L$, collision frequency $v$, electron density $n_{\mathrm{e}}$ and wave working frequency $f$ in a $\phi 800 \mathrm{~mm}$ high temperature shock tube. In experiments, $L$ is set to $4 \mathrm{~cm}$ and $38 \mathrm{~cm} . \quad v$ is $2 \mathrm{GHz}$ and $15 \mathrm{GHz}$. $n_{\mathrm{e}}$ is from $1 \times 10^{10} \mathrm{~cm}^{-3}$ to $1 \times 10^{13} \mathrm{~cm}^{-3}$, and $f$ is set to $2,5,10,14.6 \mathrm{GHz}$, respectively. Meanwhile, Wentzel-Kramers-Brillouin (WKB) and finite-difference time-domain (FDTD) methods are adopted to carry out theoretical simulation for comparison with experimental results. It is found that when $L$ is much larger than EM wavelength $\lambda$ (thick sheath) and $v$ is large, the theoretical result is in good agreement with experimental one, when sheath thickness $L$ is much larger than $\lambda$, while $v$ is relatively small, two theoretical results are obviously different from the experimental ones. It means that the existing theoretical model can not fully describe the contribution of $v$. Furthermore, when $L$ and $\lambda$ are of the same order of magnitude (thin sheath), the experimental result is much smaller than the theoretical values, which indicates that the current model can not properly describe the thin sheath effect on EM attenuation characteristics.
\end{abstract}

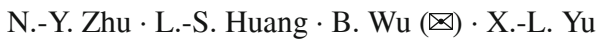
State Key Lab of High Temperature Gas Dynamics (LHD), Institute of Mechanics, Chinese Academy of Sciences, 100190 Beijing, China

e-mail:wubin@imech.ac.cn

R.-H. Wu · G. Meng · A.-M. Ren · X.-T. Hu

State Key Lab of Experimental Physics

and Computational Mathematics,

China Aerospace Technology Corporation,

100076 Beijing, China
Keywords Plasma - Electromagnetic wave - Shock tube Numerical simulation

\section{Introduction}

The transmission of electromagnetic (EM) wave in an infinite uniform plasma without external magnetic field is a well-posed problem with well-defined theoretical model [13]. However, actual plasmas met in engineering applications are finite in length and non-uniform, even extremely nonuniform. For example, the plasma sheath around the re-entry vehicle generated by high temperature high speed flow is in such condition and it can always causes intensity attenuation, even interruption, of EM wave transmission. Therefore, communication between the vehicle and the ground is often interrupted or blocked during the trajectory of a reentry hypersonic vehicle. For the plasma sheath around the re-entry vehicle, the sheath thickness is rather different under different flight conditions, and the plasma is non-uniform. There are still no rigorous and precise theoretical estimation of EM wave transmission characteristics for this kind of sheath, and it is necessary to carry out ground simulation experiments to study the specific characteristics, and thus theoretical and experimental studies should be reinforced in this field $[4,5]$. In our previous studies we have preliminarily observed some specific phenomenon of wave transmission attenuation in thin plasma sheath [4-6]. Recently, the weakly ionized plasma behind shock wave has been used to simulate flight conditions by using $\phi 800 \mathrm{~mm}$ high temperature shock, and systematic studies on the characteristics of EM wave transmission coefficient $T$ versus plasma sheath thickness $L$, collision frequency $v$, electron density $n_{\mathrm{e}}$ and microwave working frequency $f$ have been carried out. Regular variable parameters are obtained and important results are accomplished in the experiments. The experimental results are compared with the calculation using two methods commonly adopted in engineering practice, i.e., Wentzel-Kramers-Brillouin (WKB) and finite-difference time-domain (FDTD), to reveal thereby their limitations. 


\section{Experimental facility}

The high temperature gas behind the strong shock wave in a $\phi 800 \mathrm{~mm}$ large shock tube is used as the high speed plasma source. Refer to Ref. [7] for detail of the structure and working characteristics of the $\phi 800 \mathrm{~mm}$ shock tube, where the operation ranges for the initial pressure and Mach number are respectively $P_{1}=1.3-133 \mathrm{~Pa}$ and $M_{\mathrm{s}}=8-23$ (i.e., the gas velocity in the range of $2.7-7.8 \mathrm{~km} / \mathrm{s}$ ). The high temperature gas behind the strong shock wave is in a relatively uniform ionized state. For air as the working medium, the electron density in Region 2 is in the range of $1 \times 10^{10}-2 \times 10^{13} \mathrm{~cm}^{-3}$, and the maximum electron density in Region 5 can be as high as $1 \times 10^{15} \mathrm{~cm}^{-3}$. The length of the high speed, high temperature "gas piston" varied from dozens of centimeters to more than $1 \mathrm{~m}$. Since the shock tube has good repeatability and controllability, we can control the initial pressure $P_{1}$ and Mach number $M_{\mathrm{s}}$ to get the desired electron density $n_{\mathrm{e}}$ and collision frequency $v$.

By selecting diaphram with proper thickness and changing the mixture ratios between hydrogen, oxygen and nitrogen in the high pressure chamber, we can make the operation gas velocity in the shock tube close to the desired value. By means of the vacuum system and distribution system for the low pressure chamber, we can make the initial pressure in the experimental section close to the ambient air density at the specific flight height. The shock velocity can be deduced from multiple ionization probes located on the shock tube side wall via data acquisition unit and storage oscilloscope. The flow field is monitored by optical radiation measurement system, and side wall electric probes.

Through controlling the width of experimental section for EM wave transmission which was attached to the end section of the shock tube, desired sheath thickness of plasma can be set as $4 \mathrm{~cm}, 38 \mathrm{~cm}$ or others. The detailed configuration is shown in Fig. 1.

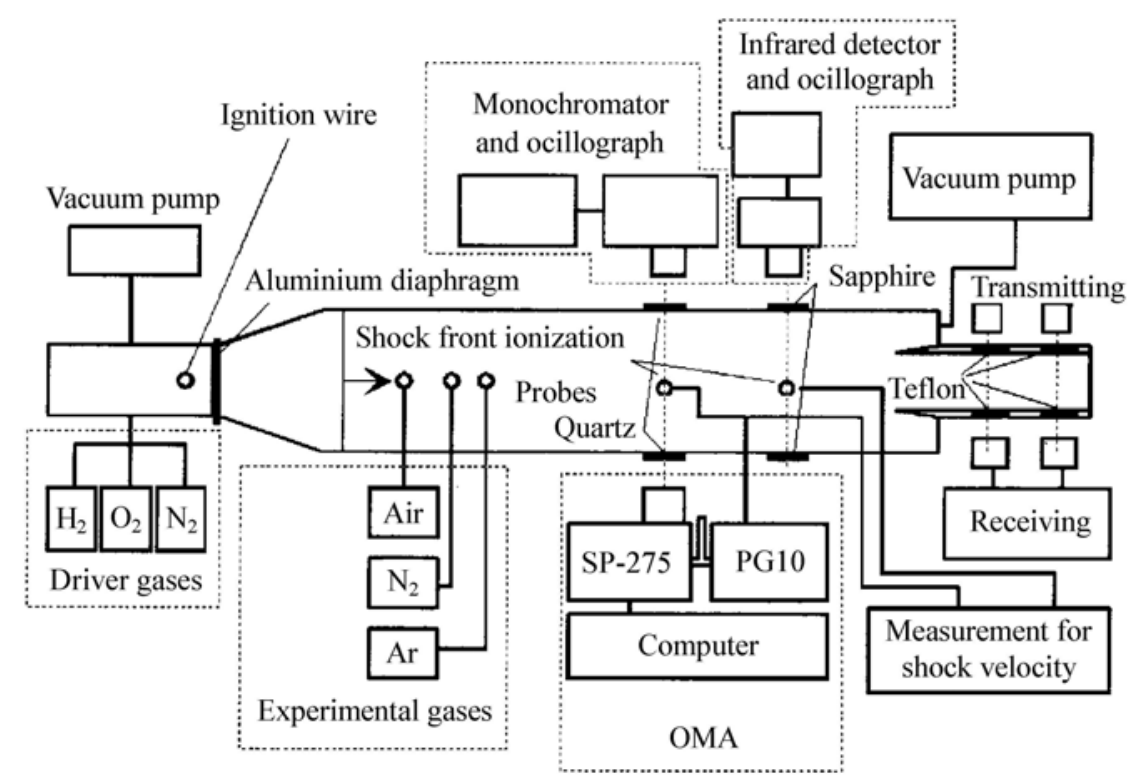

Fig. 1 Schematic diagram of $\phi 800 \mathrm{~mm}$ shock tube and instrumentation arrangement

A Network Analyzer (Model: PNA E8362B, Agilent Technologies ) is used for EM generation with the frequency set from $10 \mathrm{MHz}$ to $20 \mathrm{GHz}$. Since the effective time period for each measurement of the maximum electron density after the normal shock wave is only dozens of microseconds, normally a certain fixed frequency is selected for each measurement.

Once the initial pressure and Mach number in the shock tube are measured, the electron density in Region 2 can be obtained from theoretical or experimental charts [7].

Theoretical and experimental studies on the collision frequency in high temperature gas flow carried out jointly by the Institute of Mechanics, the Institute of Physics and the State Lab of Experimental Physics and Computational
Mathematics from 2005 to 2008 have acquired satisfactory achievements, and the total collision frequency can thus be quite accurately derived from given temperature, density and compositions in a high temperature high speed flow field.

\section{Numerical simulation for EM wave propagation in a shock wave}

Existence of boundary layer in a shock tube will lead to non-uniform plasma parameters distributions. We propose two theoretical calculation methods to estimate transmission characteristics of EM wave in a non-uniform plasma. The boundary effect was estimated by a method proposed in Ref. [8]. 


\subsection{Numerical simulation I: WKB method}

For studying the absorption, reflection and transmission of electromagnetic waves in plasmas, the following basic assumptions are made for the plasma medium. The plasma is an infinite plate with 1-dimensional distribution, i.e., the plasma characteristics are variable only in the transmission direction [9].

When an electromagnetic wave is propagating in a plasma, its transmission characteristics are dependent on the plasma's dielectric constant $\varepsilon$ and conductivity $\sigma$, and can, by referring to plasma theory $[1,3]$, be expressed as

$\varepsilon=\varepsilon_{0}-\frac{n_{\mathrm{e}} e^{2}}{m_{\mathrm{e}}} \frac{1}{\omega^{2}+v^{2}}$,

$\sigma=\frac{n_{\mathrm{e}} e^{2}}{m_{\mathrm{e}}} \frac{v}{\omega^{2}+v^{2}}$

The relative complex dielectric constant of the plasma is

$\varepsilon_{\mathrm{r}}=\left(1-\frac{\omega_{\mathrm{p}}^{2}}{\omega^{2}+v^{2}}\right)+\mathrm{i} \frac{\omega_{\mathrm{p}}^{2}}{\omega^{2}+v^{2}} \frac{v}{\omega}$,

where $\omega_{\mathrm{p}}$ is the plasma's circular frequency described as follows

$\omega_{\mathrm{p}}=\sqrt{\frac{n_{\mathrm{e}} e^{2}}{m_{\mathrm{e}} \varepsilon_{0}}}$.

Then, the reflection coefficient $\mu$ and the decay coefficient $\chi$ are respectively

$$
\begin{aligned}
\mu= & \operatorname{Re}\left(\sqrt{\varepsilon_{\mathrm{r}}}\right)=K_{\mathrm{r}} \\
= & \left\{\frac{1}{2}\left(1-\frac{\omega_{\mathrm{p}}^{2}}{\omega^{2}+v^{2}}\right)\right. \\
& \left.+\frac{1}{2}\left[\left(1-\frac{\omega_{\mathrm{p}}^{2}}{\omega^{2}+v^{2}}\right)^{2}+\left(\frac{\omega_{\mathrm{p}}^{2}}{\omega^{2}+v^{2}} \frac{v}{\omega}\right)^{2}\right]^{\frac{1}{2}}\right\}^{\frac{1}{2}}, \\
\chi= & -\operatorname{Im}\left(\sqrt{\varepsilon_{\mathrm{r}}}\right)=K_{\mathrm{i}} \\
= & -\frac{1}{2}\left(1-\frac{\omega_{\mathrm{p}}^{2}}{\omega^{2}+v^{2}}\right) \\
& \left.+\frac{1}{2}\left[\left(1-\frac{\omega_{\mathrm{p}}^{2}}{\omega^{2}+v^{2}}\right)^{2}+\left(\frac{\omega_{\mathrm{p}}^{2}}{\omega^{2}+v^{2}} \frac{v}{\omega}\right)^{2}\right]^{\frac{1}{2}}\right\}^{\frac{1}{2}} .
\end{aligned}
$$

\subsection{Numerical simulation II: FDTD method}

Generally, EM wave transmission characteristics can be given by Maxwell Equations, which describe all the basic rules of macroscopic EM fields, whereas FDTD is a method using finite difference method to solve Maxwell Equations in the time domain.
Principle in the application of FDTD method is as follows [10-15]. Yee discretation format is adopted to sample alternatively in the space and time directions all the components of $\mathrm{E}$ and $\mathrm{H}$ fields in Maxwell Equations.

In 1D FDTD simulation $[10,11]$, the principle for calculating the reflection and the transmission coefficients for EM wave propagation in plasma is shown in Fig. 2. The problem of EM wave incidence on 1D plasma must be abstracted into a 1D model and be expressed onto mesh via FDTD method [10]. In computation there are two problems needed treatment as follows. First, the planar wave is introduced. Then, we adopt a so-called "isolating boundary" technique to set an "isolating boundary" at the nodal point of $k k_{0}$, where the incident wave is allowed to propagate to the right side only, however, the left-going transmission wave at the plasma interface is allowed to pass through the isolation boundary. Hence there is only reflection wave being sampled at the nodal point of $k_{1}$ and there is only transmission wave being sampled at the nodal point of $k_{2}$. Second, the absorption boundary is set. When the reflection wave and transmission wave are propagating to both ends of the simulation space, if reflection occurs at the end point, the reflection wave must be sampled by the sampler located at the nodal point of $k_{1}$ or $k_{2}$, affecting thus the accuracy or correctness of the results. Therefore, we must set absorption boundaries at both ends to make the wave propagating toward the end generate no reflection wave. In actual simulations, most adopted absorption boundary is the "perfectly matched layer" (PML) proposed by Berenger [10], where PML consists of electrical conducting medium with damping and magnetically permissible properties, and any transmission wave will be rapidly damped out there. Even for a PML with finite thickness, it will perfectly absorbed incident waves. Theoretically, incident wave with any incident angle and any frequency generates no reflection on a PML absorption boundary. PML can be somewhat viewed as wave absorption materials in darkrooms.

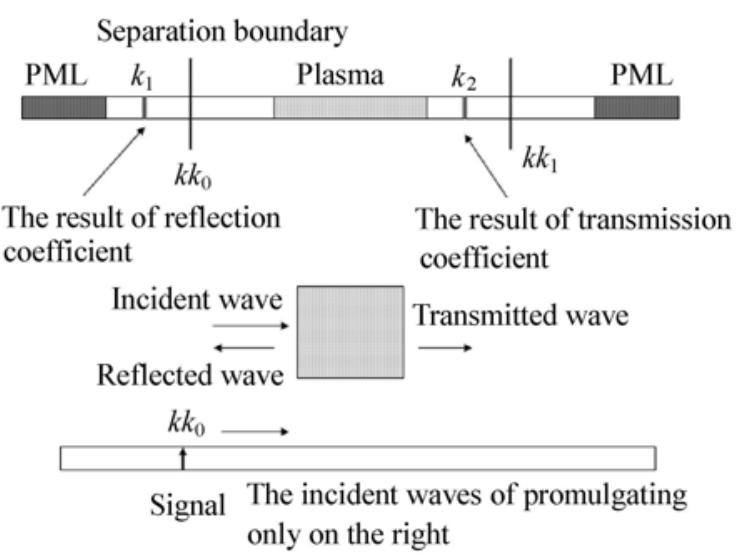

Fig. 2 1D FDTD simulation model for EM wave transmission in plasma 
By referring to the simulation model shown in Fig. 2, the following reflection coefficient $R$ and transmission coefficient $T$ can be given for EM wave transmission in plasma

$R=10 \lg \frac{P_{\mathrm{r}}}{P_{\mathrm{i}}}$

$T=10 \lg \frac{P_{\mathrm{t}}}{P_{\mathrm{i}}}$

where, $P_{\mathrm{i}}$ is power of the incident EM wave, $P_{\mathrm{r}}$ is power of the reflection EM wave, and $P_{\mathrm{t}}$ is power of the transmission EM wave.
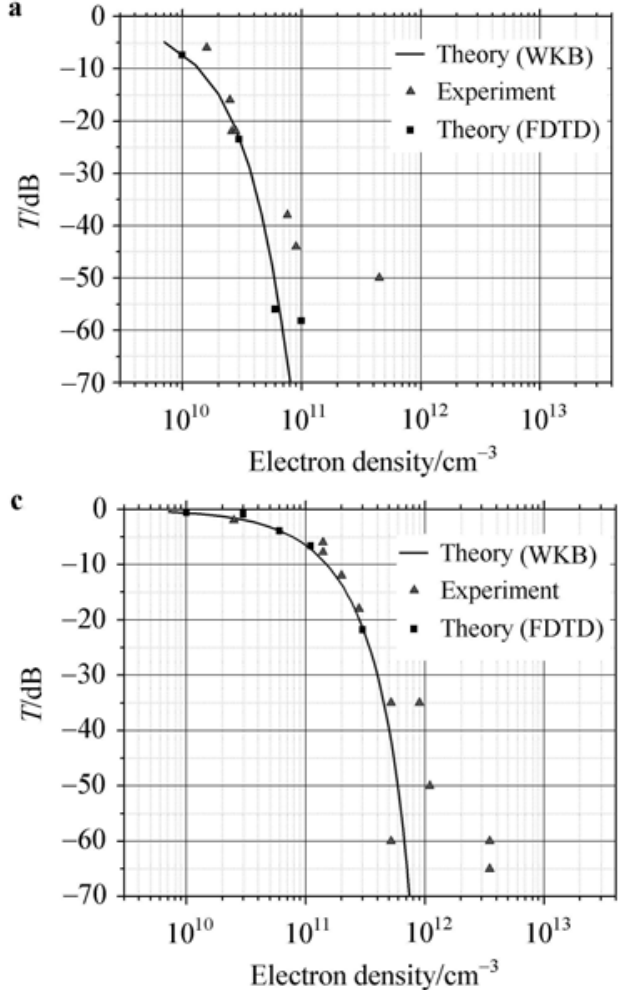

\section{Results and discussion}

4.1 Case I: Both plasma thickness $L$ and collision frequency $v$ are large

Figure 3 gives the experimental and theoretical results for electromagnetic wave transmission coefficient $T$ versus electron density $n_{\mathrm{e}}$. Here, plasma sheath thickness $L$ is $38 \mathrm{~cm}$, collision frequency $v$ is $15 \mathrm{GHz}$. The working frequency is 2, 5, 10 and $14.6 \mathrm{GHz}$, respectively. The results show that the transmission coefficient decreases with electron density and working frequency. It is found that the experiments are in agreement with the existent theoretical results.
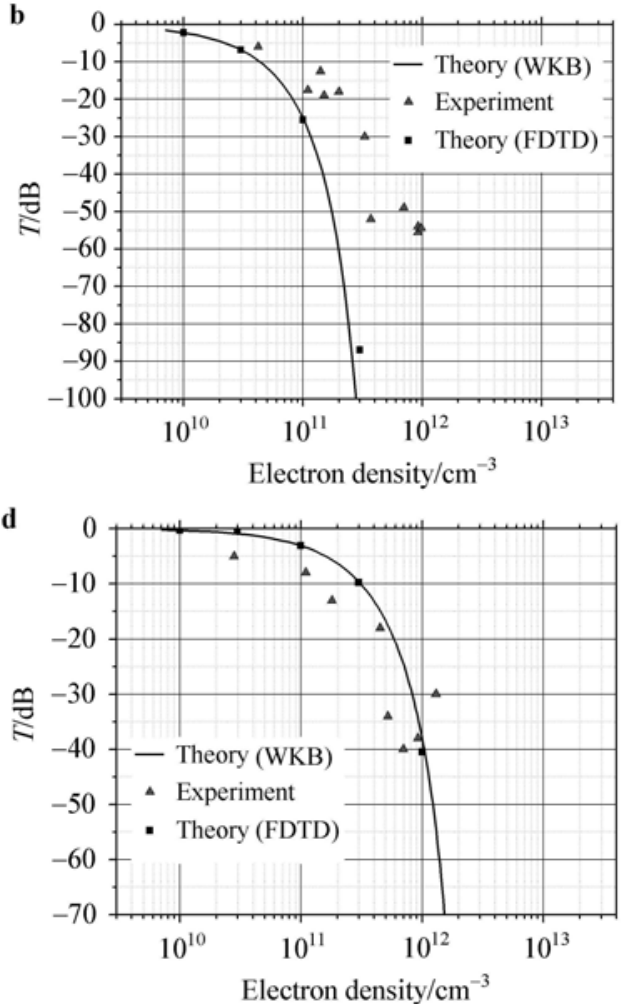

Fig. 3 The experimental and theoretical results for electromagnetic wave transmission coefficient $T$ versus electron density $n_{\mathrm{e}}$ for different working frequency (plasma sheath thickness $L=38 \mathrm{~cm}$, collision frequency $v=15 \mathrm{GHz}$, working frequency $\mathbf{a} f=2 \mathrm{GHz}, \mathbf{b} f=5 \mathrm{GHz}$, c $f=10 \mathrm{GHz}$, and $\mathbf{d} f=14.6 \mathrm{GHz})$

4.2 Case II: Plasma thickness $L$ is relatively large and collision frequency $v$ is relatively small

Figure 4 gives the experimental and theoretical results for electromagnetic wave transmission coefficient $T$ versus electron density $n_{\mathrm{e}}$. Here, plasma sheath thickness $L$ is $38 \mathrm{~cm}$, collision frequency $v$ is $2 \mathrm{GHz}$. The working frequency is 2, 5, 10 and $14.6 \mathrm{GHz}$, respectively. The results show that the transmission coefficient decreases with electron density and working frequency. But the experimental results are evidently smaller than the theoretical values.
4.3 Case III: Plasma thickness $L$ is relatively small and collision frequency $v$ is relatively large

Figure 5 gives the experimental and theoretical results for electromagnetic wave transmission coefficient $T$ versus electron density $n_{\mathrm{e}}$. Here, plasma sheath thickness $L$ is $4 \mathrm{~cm}$, collision frequency $v$ is $15 \mathrm{GHz}$. The working frequency is 2, 5, 10 and $14.6 \mathrm{GHz}$, respectively. The results show that the transmission coefficient decreases with electron density and working frequency. But the experimental results are also evidently smaller than the theoretical values. 


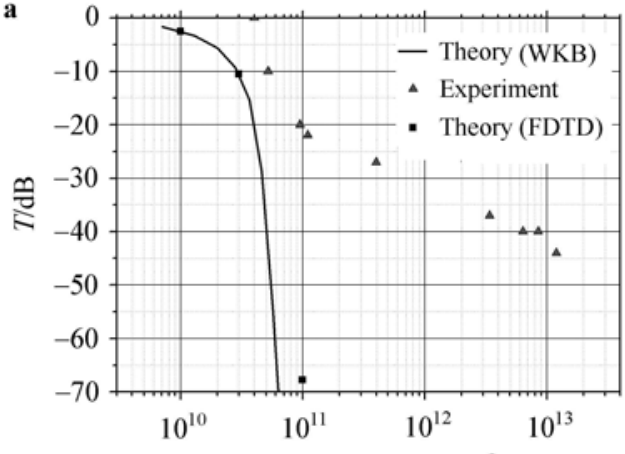

Electron density $/ \mathrm{cm}^{-3}$

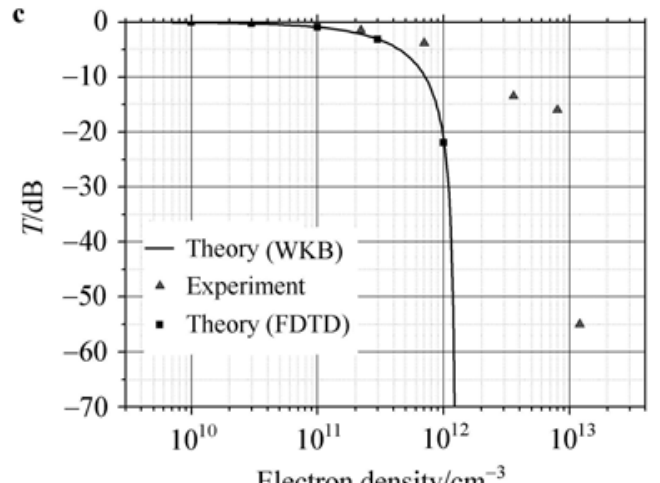

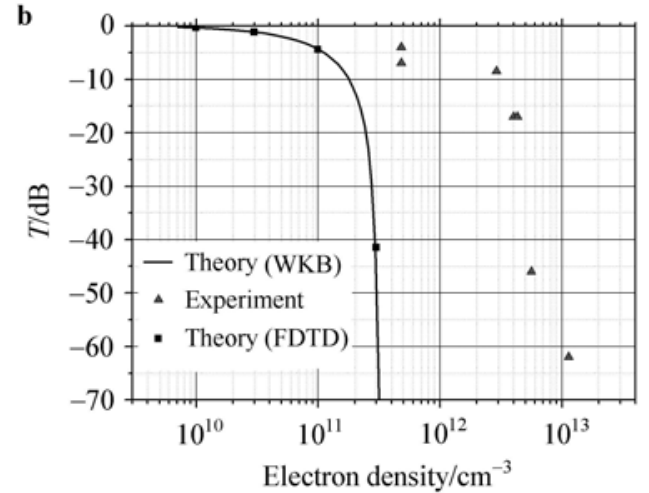

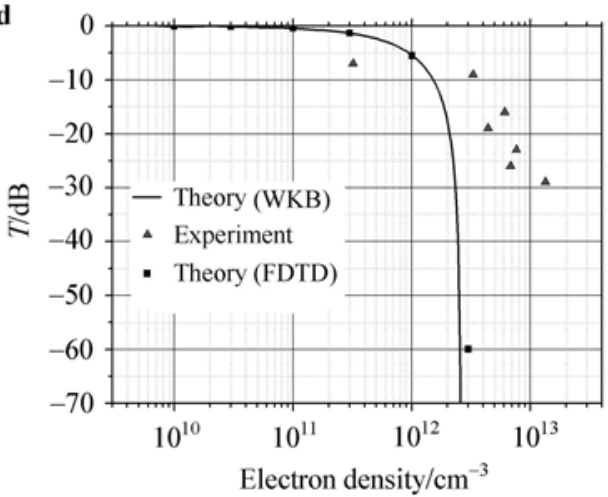

Fig. 4 The experimental and theoretical results for electromagnetic wave transmission coefficient $T$ versus electron density $n_{\mathrm{e}}$ for different working frequency (plasma sheath thickness $L=38 \mathrm{~cm}$, collision frequency $v=2 \mathrm{GHz}$, working frequency a $f=2 \mathrm{GHz}, \mathbf{b} f=5 \mathrm{GHz}$, c $f=10 \mathrm{GHz}$, and $\mathbf{d} f=14.6 \mathrm{GHz})$
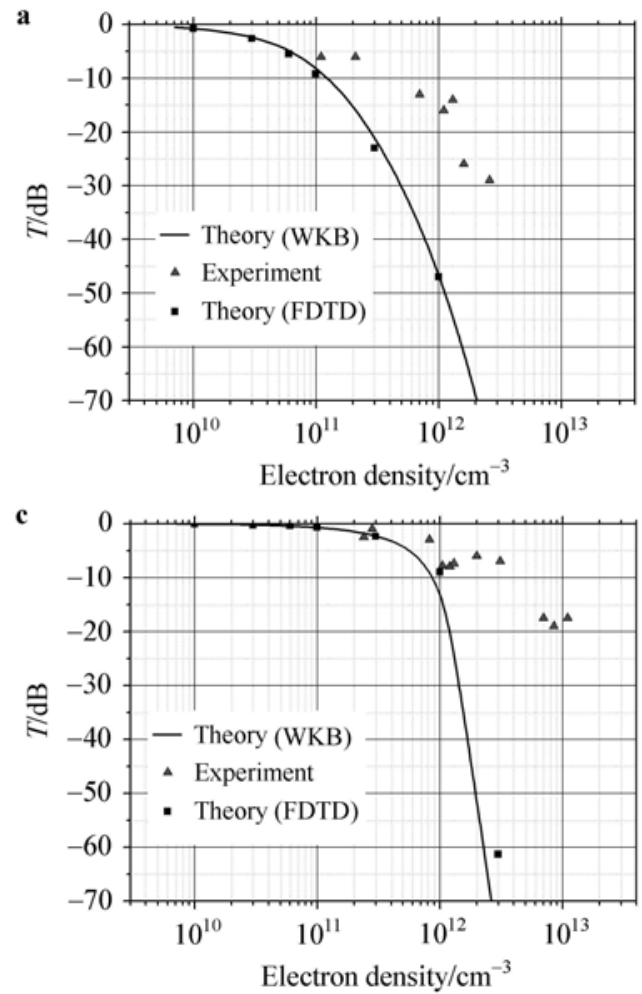
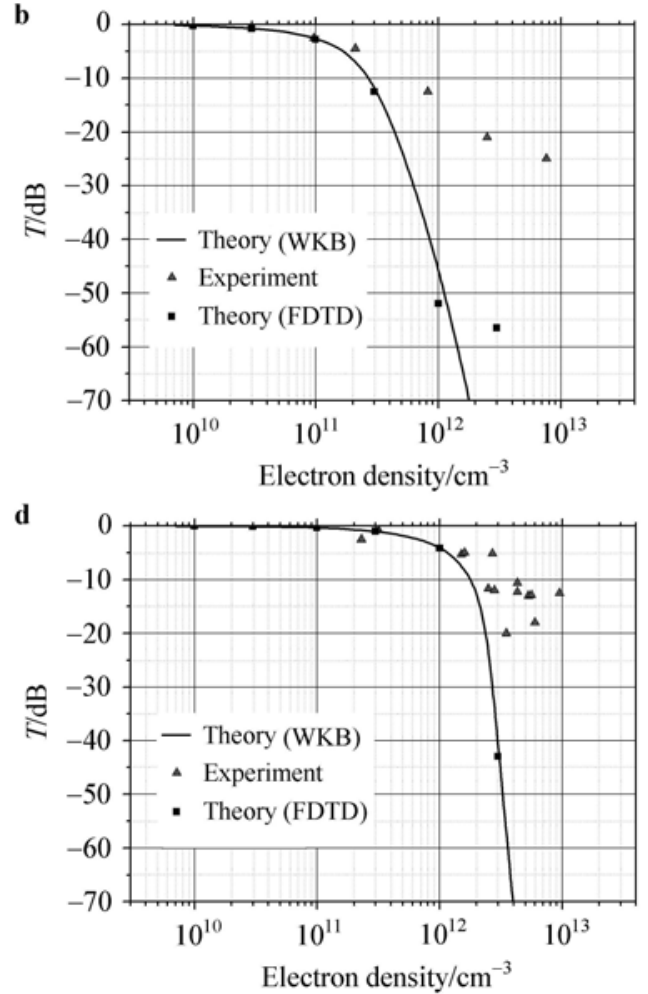

Fig. 5 The experimental and theoretical results for electromagnetic wave transmission coefficient $T$ versus electron density $n_{\mathrm{e}}$ for different working frequency (plasma sheath thickness $L=4 \mathrm{~cm}$, collision frequency $v=15 \mathrm{GHz}$, working frequency $\mathbf{a} f=2 \mathrm{GHz}, \mathbf{b} f=5 \mathrm{GHz}$, c $f=10 \mathrm{GHz}$, and $\mathbf{d} f=14.6 \mathrm{GHz}$ ) 
4.4 Case IV: Plasma thickness $L$ is relatively small and collision frequency $v$ is also relatively small

Figure 6 gives the experimental and theoretical results for electromagnetic wave transmission coefficient $T$ versus electron density $n_{\mathrm{e}}$. Here, plasma sheath thickness $L$ is $4 \mathrm{~cm}$,
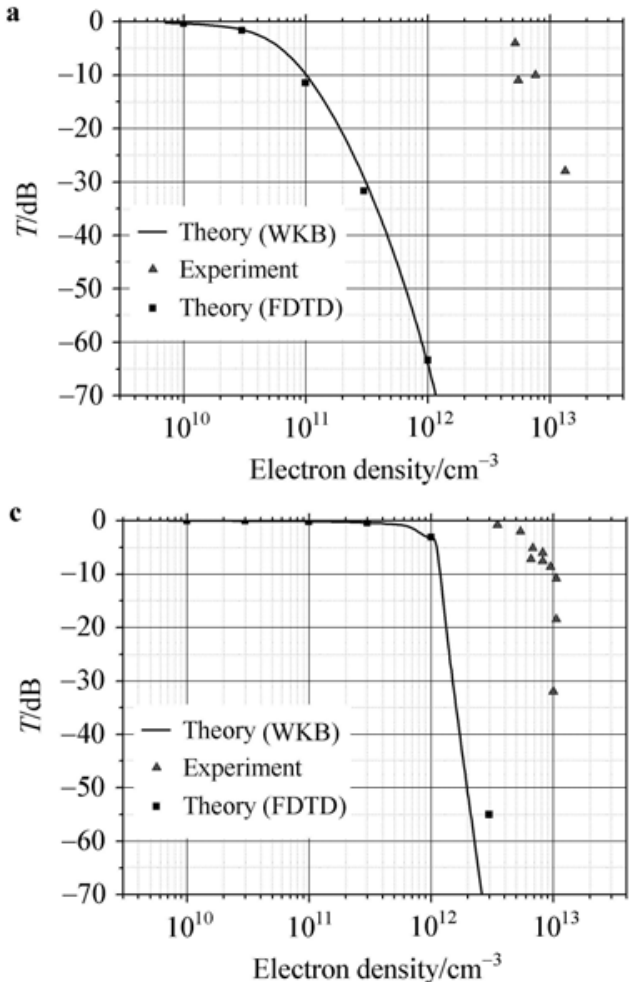

collision frequency $v$ is $2 \mathrm{GHz}$. The working frequency is 2 , 5, 10 and $14.6 \mathrm{GHz}$, respectively. The results show that the transmission coefficient decreases with electron density and working frequency. But the experimental results are much smaller than the theoretical values.
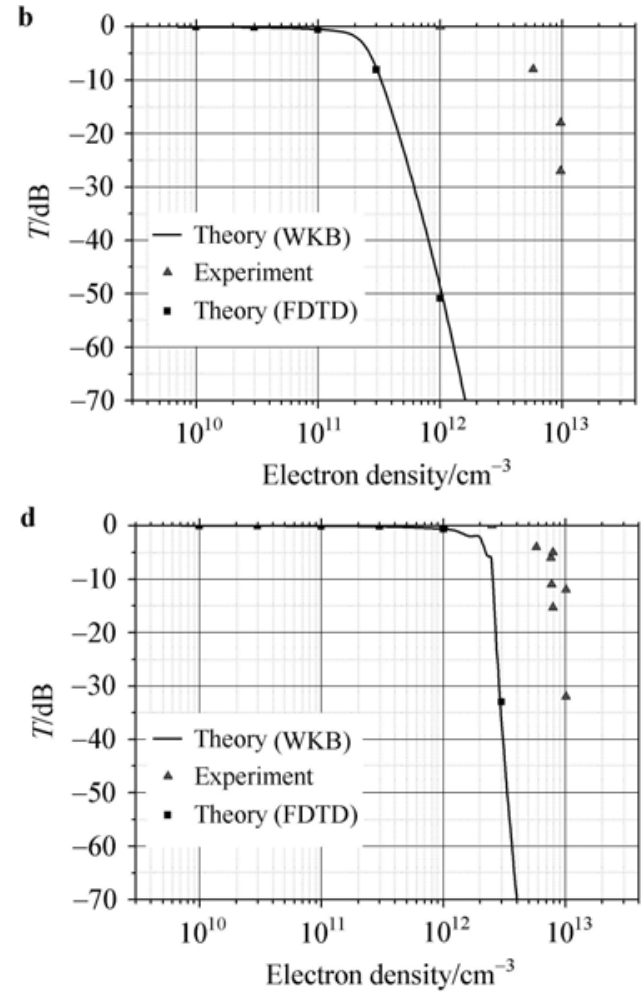

Fig. 6 The experimental and theoretical results for electromagnetic wave transmission coefficient $T$ versus electron density $n_{\mathrm{e}}$ for different working frequency (plasma sheath thickness $L=4 \mathrm{~cm}$, collision frequency $v=2 \mathrm{GHz}$, working frequency a $f=2 \mathrm{GHz}, \mathbf{b} f=5 \mathrm{GHz}$, c $f=10 \mathrm{GHz}$, and $\mathbf{d} f=14.6 \mathrm{GHz})$

\section{Conclusions}

Studies on the variations of wave attenuation characteristics versus plasma sheath thickness $L$, collision frequency $v$, electron density $n_{\mathrm{e}}$ and wave working frequency $f$ in a $\phi 800 \mathrm{~mm}$ high temperature shock tube are presents in the paper. In the test, $L$ is set to $4 \mathrm{~cm}$ and $38 \mathrm{~cm} . v$ is $2 \mathrm{GHz}$ and $15 \mathrm{GHz}$. $n_{\mathrm{e}}$ is from $1 \times 10^{10} \mathrm{~cm}^{-3}$ to $1 \times 10^{13} \mathrm{~cm}^{-3}$, and $f$ is set to $2,5,10,14.6 \mathrm{GHz}$, respectively. Meanwhile, WKB and FDTD methods are adopted to carry out theoretical simulation for comparison with experimental results. The following facts are found.

(1) When sheath thickness $L$ is much larger than EM wavelength $\lambda$ (thick sheath) and $v$ is large, the simulation and experiment results are found in good agreement. Two available theoretical methods, WKB and FDTD, have no essential difference under the present experimental condition of relatively uniform plasma.

(2) When sheath thickness $L$ is much larger than EM wave- length $\lambda$ (thick sheath), while $v$ is relatively small, two theoretical results are obviously different from the experimental ones. It indicates that the existent theories can not fully manifest the contribution of $v$.

(3) When sheath thickness $L$ and EM wavelength $\lambda$ are of the same order of magnitude (thin sheath plasma), the experimental results are much smaller than the theoretical values, indicating that both theories can not properly describe the thin sheath effect.

Acknowledgement Bing-Cheng Fan, Qun Pu, Qian-Suo Yang, Fei Li, Tian-Xiang Huang, Hong-Hao Hu, Jin-Hu Liang from the Institute of Mechanics have helped or participated in part of the experiments, hereby the authors would like to express sincere thanks.

\section{References}

1 Aucilo, O., Flamm, D.L.: Plasma Diagnostics. Academic Press, Salt Lake City (1989) 
2 Heald, M.A., Wharton, C.B.: Plasma Diagnostics with Microwaves. Jehn Wiley and Sons, New York (1965)

3 Tarit, K.B.: High Temperature Gas Dynamics, An Introduction For Physicists and Engineers. Springer, German (2004)

4 Zhu, N.Y., Li, X.F., Huang, L.S., et al.: An investigation of electromagnetic wave propagation in plasma by shock tube. Acta Mechanica Sinica 20, 212-218 (2004)

5 Wang, B.Y., Xu, Y.H., Ji, Z.Y.: Propagation of electromagnetic waves in in-homogenous and lossy reentry plasma sheath layer. Journal of Astronautics 6, 35-46 (1985) (in Chinese)

6 Zeng, X.J., Ma, P., Yu, Z.F., et al.: Experimental investigation and analysis on jet-plasma stealth in air surroundings. Journal of Experiments in Fluid Mechanics 22, 49-54 (2008) (in Chinese)

7 Zhu, N.Y., Li, X.F.: Mesurements of electron density in high temperature air and inspections of theoretical charts. Journal of Astronautics 8, 86-92 (1987) (in Chinese)

8 Mirels, H.: Turbulent boundary layer behind constant velocity shock including wall blowing effects. AIAA Journal 22, 10421047 (1984)

$9 \mathrm{Wu}, \mathrm{B} ., \mathrm{Lin}, \mathrm{L} ., \mathrm{Wu}, \mathrm{C} . \mathrm{K}$., et al.: The propagation characteristics of a vertical incidence microwave in a non-equilibrium plasma slab at atmospheric pressure. High Power Laser and Particle Beams 17, 225-228 (2005) (in Chinese)

10 Berenger, J.P.: A perfectly matched layer for the absorption of electromagnetic waves. J. Comput. Phys. 114, 185-200 (1994)

11 Ge, D.B., Yan, Y.B.: Finite Difference Time Domain Method for EM Wave. Xidian University Press, Xi' an (2002) (in Chinese)

12 Yee, K.S.: Numerical solution of initial boundary value problem involving Maxwell's equations in isotropic media. IEEE Trans Antennas Propagate 14, 302-307 (1966)

13 Zheng, Y.G.: Study of electromagnetic scattering of plasma. [M.S. Thesis]. Science of Xidian University, Xi' an (2008) (in Chinese)

14 Mo, J.J.: Study on wideband electromagnetic characteristics of stealth targets in low frequency. [Ph.D. Thesis], National University of Defense Technology, Changsha, Hunan (2004) (in Chinese)

15 Lan, C.H., Hu, X.W., Zhong, H.H., et al.: Numerical solutions of the propagation of electromagnetic wave as signal in atmospheric environment with weakly ionized plasma. In: Proceedings of Information Technology and Environmental System Sciences: ITESS 4, 489-491 (2008) 